\title{
SUPERNOVA PTF 09UJ: A POSSIBLE SHOCK BREAKOUT FROM A DENSE CIRCUMSTELLAR WIND
}

\author{
E. O. OfeK ${ }^{1,11}$, I. Rabinak ${ }^{2}$, J. D. Neill ${ }^{1}$, I. Arcavi² ${ }^{2}$, S. B. Cenko ${ }^{3}$, E. Waxman ${ }^{2}$, S. R. Kulkarni ${ }^{1}$, A. Gal-Yam ${ }^{2}$,

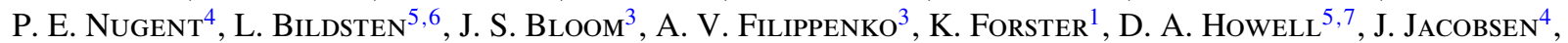 \\ M. M. Kasliwal ${ }^{1}$, N. LaW ${ }^{1,8}$, C. Martin ${ }^{1}$, D. Poznanski ${ }^{3,4,11}$, R. M. Quimby ${ }^{1}$, K. J. Shen ${ }^{5}$, M. Sullivan ${ }^{9}$, R. DeKany $^{10}$,

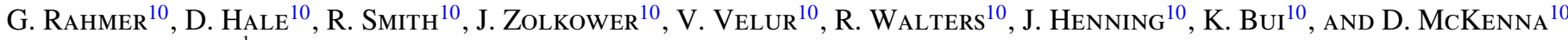 \\ ${ }^{1}$ Division of Physics, Mathematics and Astronomy, California Institute of Technology, Pasadena, CA 91125, USA \\ ${ }^{2}$ Benoziyo Center for Astrophysics, Weizmann Institute of Science, 76100 Rehovot, Israel \\ ${ }^{3}$ Department of Astronomy, University of California, Berkeley, CA 94720-3411, USA \\ ${ }^{4}$ Lawrence Berkeley National Laboratory, 1 Cyclotron Road, Berkeley, CA 94720, USA \\ ${ }^{5}$ Department of Physics, Broida Hall, University of California, Santa Barbara, CA 93106, USA \\ ${ }^{6}$ Kavli Institute for Theoretical Physics, Kohn Hall, University of California, Santa Barbara, CA 93106, USA \\ ${ }^{7}$ Las Cumbres Observatory Global Telescope Network, 6740 Cortona Dr., Suite 102, Goleta, CA 93117, USA \\ ${ }^{8}$ Dunlap Institute for Astronomy and Astrophysics, University of Toronto, 50 St. George Street, Toronto, Ontario M5S 3H4, Canada \\ ${ }^{9}$ Department of Physics, University of Oxford, Denys Wilkinson Building, Keble Road, Oxford OX1 3RH, UK \\ ${ }^{10}$ Caltech Optical Observatories, California Institute of Technology, Pasadena, CA 91125, USA \\ Received 2010 July 9; accepted 2010 September 27; published 2010 November 12
}

\begin{abstract}
Type-IIn supernovae (SNe IIn), which are characterized by strong interaction of their ejecta with the surrounding circumstellar matter (CSM), provide a unique opportunity to study the mass-loss history of massive stars shortly before their explosive death. We present the discovery and follow-up observations of an SN IIn, PTF 09uj, detected by the Palomar Transient Factory (PTF). Serendipitous observations by Galaxy Evolution Explorer (GALEX) at ultraviolet (UV) wavelengths detected the rise of the SN light curve prior to the PTF discovery. The UV light curve of the SN rose fast, with a timescale of a few days, to a UV absolute AB magnitude of about - 19.5. Modeling our observations, we suggest that the fast rise of the UV light curve is due to the breakout of the SN shock through the dense CSM $\left(n \approx 10^{10} \mathrm{~cm}^{-3}\right)$. Furthermore, we find that prior to the explosion the progenitor went through a phase of high massloss rate $\left(\sim 0.1 M_{\odot} \mathrm{yr}^{-1}\right)$ that lasted for a few years. The decay rate of this SN was fast relative to that of other SNe IIn.
\end{abstract}

Key words: stars: mass-loss - supernovae: general - supernovae: individual (PTF 09uj)

Online-only material: color figures

\section{INTRODUCTION}

Early ultraviolet (UV) detection of supernovae (SNe) of all types in the shock-breakout phase holds great potential for probing the nature and properties of SN progenitors (e.g., Colgate 1974; Falk \& Arnett 1977; Klein \& Chevalier 1978; Matzner \& McKee 1999; Waxman et al. 2007; Katz et al. 2010; Piro et al. 2010; Rabinak \& Waxman 2010; Nakar \& Sari 2010). Shock-breakout observations provide a measure of the progenitor radius and the structure of its outer layers (e.g., Soderberg et al. 2008; Gezari et al. 2008; Schawinski et al. 2008). Type-IIn SNe are characterized by the presence of a blue continuum and narrow emission lines in their optical spectra (e.g., Schlegel 1990; Filippenko 1997). These features are usually interpreted as the signatures of interaction of the SN ejecta with dense circumstellar matter (CSM), due to stellar mass loss prior to the explosion. SNe IIn are probably an inhomogeneous class of objects whose appearance is dictated by the presence of dense CSM rather than by the details of the explosion. Therefore, SNe showing evidence for strong interaction are unique probes of scenarios in which a stellar explosion follows a major mass-loss event, and they can be used to study the mass-loss rate from progenitor stars (e.g., Chugai \& Danziger 1994; Chugai et al. 1995; Fransson et al. 2002; Gal-Yam et al. 2007; Ofek et al. 2007; Smith et al. 2007; Smith \& McCray 2007; Gal-Yam \& Leonard 2009; Dessart et al. 2009).

\footnotetext{
${ }^{11}$ Einstein Fellow.
}

Here, we present the discovery of an SN IIn, PTF 09uj, which was serendipitously observed in the UV by Galaxy Evolution Explorer (GALEX) (Martin et al. 2005) shortly after the explosion.

\section{OBSERVATIONS AND DATA REDUCTION}

SN PTF 09uj was discovered on 2009 June 23.30 (UTC dates are used throughout this paper) by the Palomar Transient Factory $^{12}$ (PTF; Law et al. 2009; Rau et al. 2009) conducted with the Oschin 48 inch Schmidt telescope (P48) at Palomar Observatory. The SN is associated with an $r=17.3 \mathrm{mag}$ galaxy, SDSS ${ }^{13}$ J142010.86+533341.9 (Figure 1) - a late-type disk galaxy at a redshift of $z=0.0650 \pm 0.0001$ (distance $d \cong 292 \mathrm{Mpc}^{14}{ }^{14}$.

\subsection{Photometry}

Follow-up photometry was obtained using the automated Palomar 60 inch telescope (Cenko et al. 2006; Table 1). As noted above, this field was observed by GALEX on several occasions (Table 1).

Photometry of PTF 09uj in the PTF and P60 images was obtained by the common point-spread function image-subtraction method (Gal-Yam et al. 2004, 2008). Estimates of uncertainties were obtained from the scatter in the magnitudes of

\footnotetext{
12 http://www.astro.caltech.edu/ptf/.

13 Sloan Digital Sky Survey; York et al. (2000).

14 We assume WMAP-5 cosmological parameters (Komatsu et al. 2009) and insignificant peculiar velocity.
} 


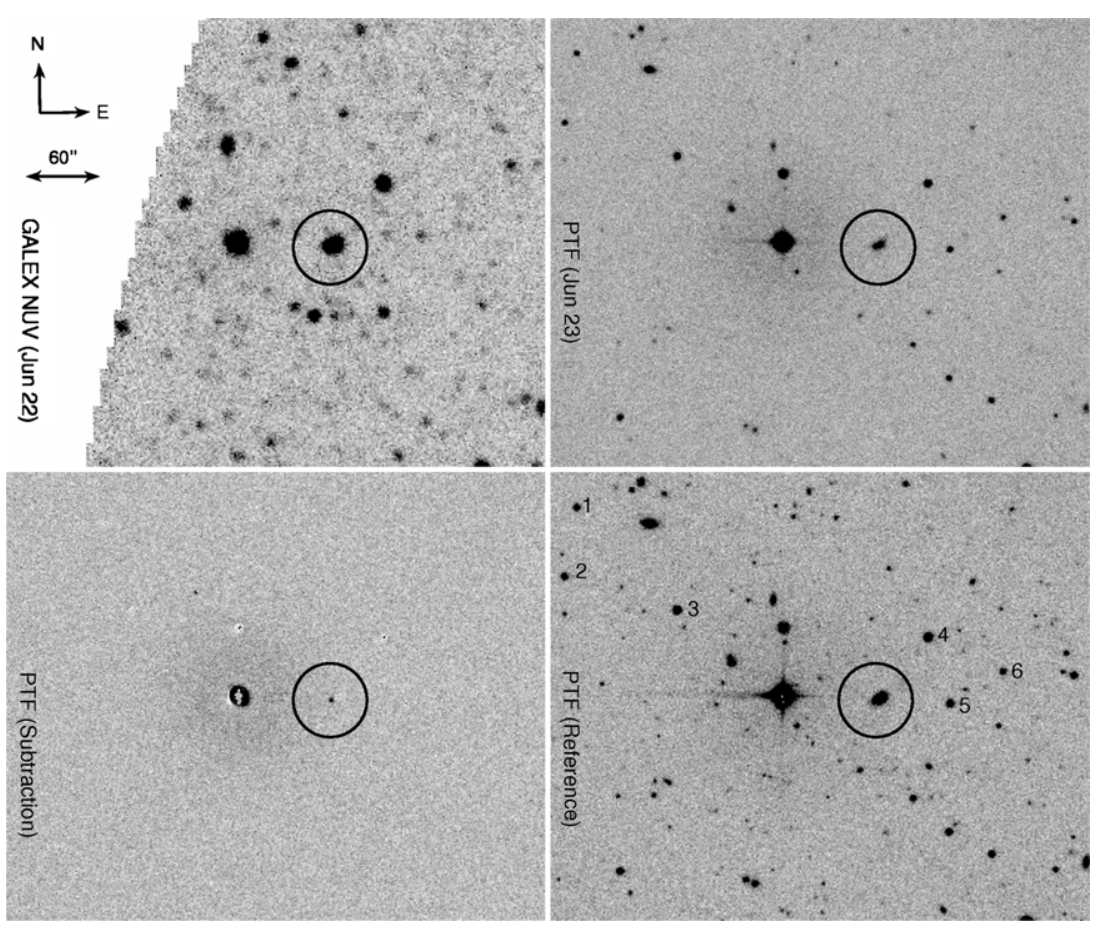

Figure 1. Images of the field of PTF 09uj. The SN was detected by GALEX in the NUV band on June 22 (upper left) and by PTF in the $R$ band on June 23 (upper right). The bottom right panel shows a reference $R$-band image prepared from PTF images obtained before the explosion. The numbered stars (1-6) mark the SDSS photometric reference stars. The bottom left panel shows the discovery of PTF 09uj using image subtraction of PTF data. A $30^{\prime \prime}$-radius circle marks the position of the $\mathrm{SN}$ in all panels. The position of the $\mathrm{SN}$ is $\alpha(\mathrm{J} 2000)=14^{\mathrm{h}} 20^{\mathrm{m}} 11^{\mathrm{s}} .15, \delta(\mathrm{J} 2000)=+53^{\circ} 33^{\prime} 41^{\prime \prime} .0$, which is $2^{\prime \prime} .7$ from the center of SDSS J142010.86+533341.9 at a position angle of $\approx 110^{\circ}$.

artificial sources. The photometry was calibrated using $r$ band magnitudes of six SDSS stars (Figure 1). Calibration errors were added in quadrature to the image-subtraction errors (Table 1).

The GALEX photometry was carried out by performing aperture photometry with a $10^{\prime \prime}$ radius around the SN host galaxy and subtracting its light as measured in the reference image. The reference image was constructed by combining the four GALEX images of this field taken prior to the SN explosion, between 2009 May 4 and May 14. Since GALEX uses photon-counting detectors (i.e., individual photons are time tagged; Martin et al. 2005), we had the opportunity to look for flux variations on relatively short timescales. In particular, we examined the earliest image in which the SN was detected, which was taken on June 22 and had an exposure time of $1364 \mathrm{~s}$. We extracted the time tags of the 4597 photons found within $10^{\prime \prime}$ of the $\mathrm{SN}$ and binned these photons on timescales from 3 to $1000 \mathrm{~s}$. We found no significant variations in flux as a function of time. We note that about $40 \%$ of these photons originate from the SN and the rest are due to the host galaxy. The GALEX NUV and P48 $R$-band light curves of PTF 09uj are presented in Figure 2.

\subsection{Spectroscopy}

We observed PTF 09uj with an exposure time of $1800 \mathrm{~s}$ with the Kast double spectrograph (Miller \& Stone 1993) mounted at the Cassegrain focus of the Shane 3-m telescope at Lick Observatory; the $5500 \AA$ dichroic was employed. On the blue arm, we used the 600 lines $\mathrm{mm}^{-1}$ grism blazed at $4310 \AA$ to provide spectral coverage of $3500-5550 \AA$ and a dispersion of $1.02 \AA \mathrm{pixel}^{-1}$, while on the red arm we used the 300 lines $\mathrm{mm}^{-1}$ grating blazed at $7500 \AA$ for a wavelength range of 5400-10000 $\AA$ and a dispersion of $4.60 \AA$ pixel $^{-1}$.

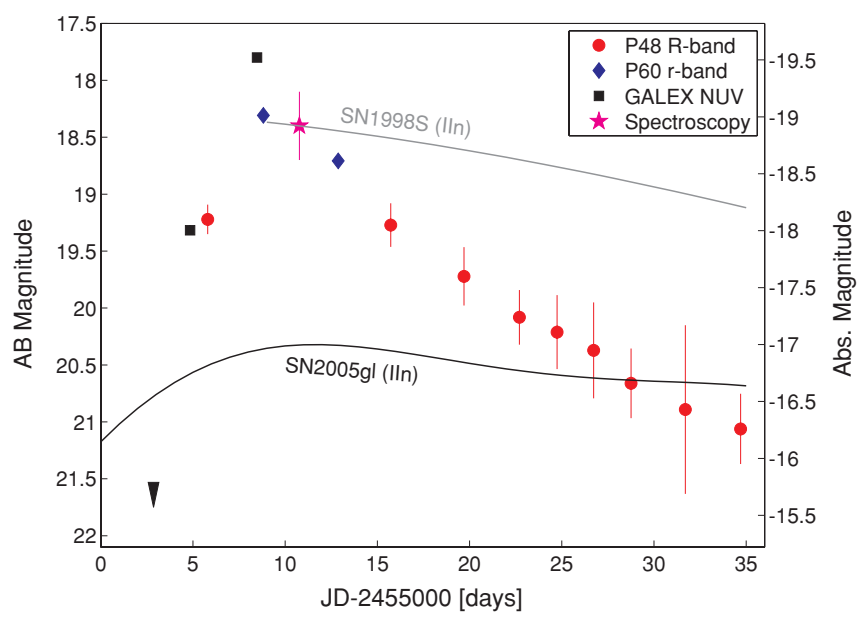

Figure 2. Light curve of PTF 09uj from the P48 (red circles), P60 (blue diamonds), synthetic photometry (magenta star), and GALEX NUV observations (black squares). The tip of the black triangle marks the magnitude lower limit from GALEX. The curves show the scaled and smoothed light curves of two other SNe IIn (SN 1998S, Fassia et al. 2000; SN 2005gl, Gal-Yam et al. 2007). (A color version of this figure is available in the online journal.)

The Lick spectrum was reduced using standard routines in $\operatorname{IRAF}^{15}$ (details provided by Cenko et al. 2008). Flux calibration was performed relative to the standard stars $\mathrm{BD}+28^{\circ} 4211$ (blue side) and $\mathrm{BD}+26^{\circ} 2606$ (red side). The Lick spectrum of PTF 09uj is shown in Figure 3. Given the lack of Na I absorption lines in the SN spectrum and the low Galactic extinction toward this SN ( $E_{B-V}=0.011 \mathrm{mag}$; Schlegel et al. 1998), we do not correct for extinction.

\footnotetext{
15 IRAF is distributed by the National Optical Astronomy Observatory, which is operated by the Association for Research in Astronomy, Inc., under cooperative agreement with the National Science Foundation.
} 
Table 1

Observations of PTF 09uj

\begin{tabular}{lcccc}
\hline \hline Telescope & UTC 2009 & Band & $\begin{array}{c}\text { Magnitude } \\
(\mathrm{AB} \text { mag })\end{array}$ & $\begin{array}{c}f_{v} \\
(\mu \mathrm{Jy})\end{array}$ \\
\hline P48 & Jun 02.3 & $R^{\mathrm{b}}$ & $>20.5$ & $<23$ \\
& Jun 23.30 & $R$ & $19.22 \pm 0.13$ & 75 \\
& Jul 03.22 & $R$ & $19.27 \pm 0.20$ & 71 \\
& Jul 07.20 & $R$ & $19.72 \pm 0.25$ & 47 \\
& Jul 10.21 & $R$ & $20.08 \pm 0.24$ & 34 \\
& Jul 12.24 & $R$ & $20.21 \pm 0.32$ & 30 \\
& Jul 14.23 & $R$ & $20.37 \pm 0.42$ & 26 \\
& Jul 16.26 & $R$ & $20.66 \pm 0.31$ & 20 \\
& Jul 19.21 & $R$ & $20.89 \pm 0.74$ & 16 \\
P60 & Jul 22.20 & $R$ & $21.06 \pm 0.30$ & 14 \\
\hline & Jun 26.31 & $g$ & $18.17 \pm 0.07$ & 197 \\
& Jun 26.31 & $r$ & $18.31 \pm 0.04$ & 173 \\
& Jun 26.31 & $i$ & $18.42 \pm 0.05$ & 159 \\
& Jun 30.38 & $g$ & $18.69 \pm 0.07$ & 122 \\
& Jun 30.38 & $r$ & $18.71 \pm 0.05$ & 119 \\
\hline GALEX & Jun 30.38 & $i$ & $18.70 \pm 0.11$ & 122 \\
\hline Lick & Jun 20.36 & NUV & $>21.7$ & $<7.5$ \\
& Jun 22.35 & NUV & $19.32 \pm 0.04$ & 67 \\
& Jun 25.98 & NUV & $17.80 \pm 0.02$ & 274 \\
\hline & Jun 28.27 & spec & & \\
& & $r$ & $18.4 \pm 0.3$ & 159 \\
\hline
\end{tabular}

Notes. $f_{v}$ is calculated at $2316 \AA, 4718 \AA, 6184 \AA$, and $7499 \AA$ for the NUV, $g$, PTF $R / r$, and $i$ bands, respectively. Magnitude uncertainties include (in quadrature) absolute calibration errors of $0.099 \mathrm{mag}$ for the PTF $R$-band measurements, and $0.071,0.037$, and $0.029 \mathrm{mag}$ for the P60 $g, r$, and $i$ bands, respectively. An aperture correction of $0.12 \mathrm{mag}$ was applied to the GALEX NUV magnitudes (Morrissey et al. 2007).

a The last P48 non-detection before the discovery.

b All of the P48 observations were conducted using the Mould $R$-band filter. Photometry was measured in the combined images of the same field taken each night (usually two).

${ }^{c}$ The magnitude from Lick observatory is based on synthetic photometry of the spectrum using the code described by Poznanski et al. (2002).

\section{INTERPRETATION}

The spectrum of PTF 09uj, which was taken around peak light, shows a blue continuum, with narrow $\mathrm{H} \alpha$ emission and no prominent, broad absorption features. This "narrow" line is actually broader than the $\mathrm{H} \alpha$ line from the host galaxy and shows a hint of a P Cygni profile (Figure 3 inset). The spectrum also exhibits a narrow He I emission line (measured rest wavelength $5884 \AA$, corresponding to He I $\lambda$ 5876) which is not present in the host-galaxy spectrum. These observations suggest that PTF 09uj is an SN IIn enshrouded with a dense CSM.

However, the $e$-folding decline rate of the $\mathrm{SN}$ flux is about 10 days. This is faster than the steepest declining SNe IIn previously known, such as SN 1998S (Fassia et al. 2000), SN 2005gl (Gal-Yam et al. 2007), and SN 2005ip (e.g., Smith et al. 2009); compare with the light curves of the first two in Figure 2.

Another possible difference between PTF 09uj and SN 1998S is the spectra. While the SNe spectra shown in Figure 3 were both taken around maximum light, PTF 09uj evolved faster and therefore these spectra probably do not correspond to the same epoch after explosion. In order to compare the spectra of the $\mathrm{SNe}$ taken at the same epoch after explosion it is probably more adequate to inspect the spectrum of SN 1998S taken 13 days prior to maximum light (Fassia et al. 2001). This earlier

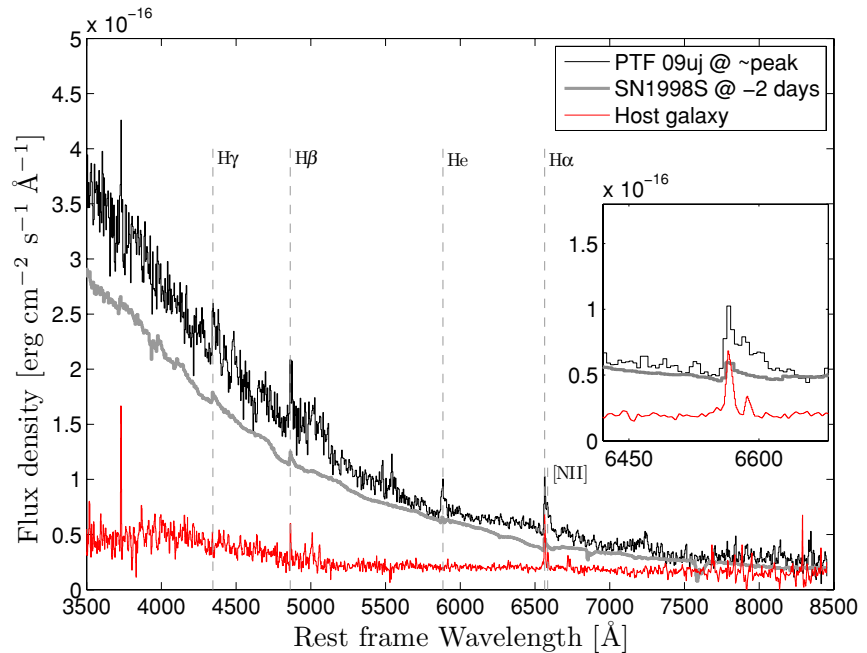

Figure 3. Spectrum of PTF 09uj (black line; upper spectrum) and its host galaxy (red line; lower spectrum). For comparison, the scaled spectrum of the Type-IIn SN 1998S (Fassia et al. 2001) two days prior to maximum light is shown just below the spectrum of PTF 09uj (gray line). The inset shows the $\mathrm{H} \alpha$ line in detail.

(A color version of this figure is available in the online journal.)

spectrum of SN 1998S is different, with broader emission lines and strong "Wolf-Rayet"-like features (e.g., C III, N III).

\subsection{Shock Breakout in a Stellar Wind}

The fast rise in UV light and the high peak luminosity $\left(v f_{v} \approx 3 \times 10^{43} \mathrm{erg} \mathrm{s}^{-1}\right)$ motivates us to consider a model of a shock breakout which takes place within a dense, optically thick, stellar wind (see also Falk \& Arnett 1977; Waxman et al. 2007). The blue continuum in the visible-light spectrum of PTF 09uj suggests that the emission is optically thick. Fitting a blackbody curve to the NUV, $g_{-}, r$-, and $i$-band photometry, obtained on June $25-26$, we find a best-fit temperature of $\approx 1.7 \times 10^{4} \mathrm{~K}$ (with $\mathrm{rms}$ of $0.13 \mathrm{mag}$ ). We note that if line blanketing is affecting the spectrum, the true effective temperature could be even higher.

In the framework of the model considered here (see sketch in Figure 4), the rising UV emission is due to a shock breakout within an optically thick wind. Some or most of the visible-light emission at later times is caused by diffusion of the shockdeposited energy.

We now calculate the properties of the shock and ejecta that are needed in order to explain the observations. In this calculation, we use the observed peak luminosity and the rise time of the $\mathrm{SN}$ to calculate various parameters (i.e., mass, velocity, temperature).

The thickness of a radiation-mediated shock, $\tau_{\mathrm{s}}$, in units of the Thomson optical depth (e.g., Weaver 1976), is given by

$$
\tau_{\mathrm{s}} \approx c / v_{\mathrm{s}} .
$$

Here, $c$ is the speed of light and $v_{\mathrm{s}}$ is the upstream ejecta (and shock) speed. A radiation-mediated shock "breaks down" or "breaks out" (i.e., radiation escapes ahead of the shock) when photons diffuse ahead of the shock faster than the shock propagates. For a wind-density profile $\rho(r)=K r^{-2}$, where $K$ is a normalization constant and $r$ is the distance from the center, the photon diffusion time from $r$ to $2 r$ is $t_{\text {diff }}=\kappa \rho r^{2} / c=\kappa K / c$, independent of $r$, while the shock propagation time is $r / v_{\mathrm{s}}$, growing with $r$. Thus, the shock breaks down when it reaches $r_{\text {break }}=\kappa K v_{\mathrm{s}} / c$. At this point, photons would diffuse and 


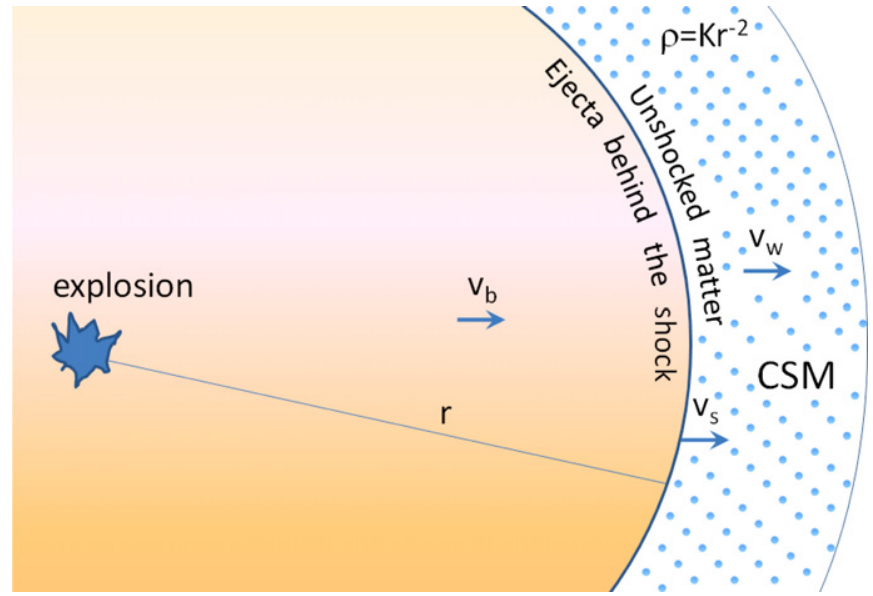

Figure 4. Sketch of our model for PTF 09uj. Fast ejecta from the SN explosion are interacting with a CSM having a wind-like density profile. Here $v_{\mathrm{w}}$ is the wind speed. See definitions of variables in Section 3.1.

(A color version of this figure is available in the online journal.)

escape the wind on a timescale equal to $t_{\text {diff }}$ multiplied by a $\log$ correction factor $\ln \left(c / v_{\mathrm{s}}\right)$.

An alternative definition of the breakout radius is the radius at which the thickness of the shock is comparable to the scale height of the density variation. Since for a wind profile this scale is $r$, with a corresponding optical depth $\tau=\kappa \rho r=\kappa K / r$, this happens at $\tau_{\mathrm{s}}=\kappa K / r=c / v_{\mathrm{s}}$ or $r_{\text {break }}=\kappa K v_{\mathrm{s}} / c$, the same as obtained above. For small radii $r$, the shock expansion timescale $\left(t_{\text {exp }}=r / v\right)$ is shorter than $t_{\text {diff }}$, and breakout takes place when $t_{\text {diff }} \approx t_{\text {exp }}$. We further note that $v_{\mathrm{s}}$ corresponds to the velocity of the faster parts of the shock. In Section 3.2 we will derive the ratio of this velocity to the bulk velocity of the ejecta $\left(v_{\mathrm{b}}\right)$.

Assuming a radiation-dominated shock, the downstream temperature of the shock can be estimated by comparing the radiation energy density (assuming blackbody radiation) with the kinetic energy per unit volume:

$$
a T^{4} \approx \frac{7}{2} \rho_{\mathrm{s}} v_{\mathrm{s}}^{2} \approx \frac{7 c}{2 t \kappa},
$$

where $a$ is the radiation constant, $t$ is the time since the explosion, $\kappa$ is the opacity, and $\rho_{\mathrm{s}}$ is the density of the stellar wind at the shock breakout. Note that the right-hand side of Equation (2) is derived assuming a wind-density profile $\rho(r) \propto r^{-2}$ along with Equation (1) and $\tau=\int_{r_{\mathrm{s}}}^{\infty} \kappa \rho(r) d r=\kappa \rho r_{\mathrm{s}}$. The factor of 7 arises from the shock's compression ratio $(\gamma+1) /(\gamma-1)=7$, with an adiabatic index of $\gamma=4 / 3$. Here $r_{\mathrm{s}}$ is the radius at which the shock breakout takes place.

In this framework, the kinetic energy of the explosion is converted into radiation at a rate of $L \approx 2 \pi r_{\mathrm{s}}^{2} v_{\mathrm{s}} \rho_{\mathrm{s}} v_{\mathrm{s}}^{2}$. Here we assume that the shock is optically thick and that the emission spectrum is roughly represented by a blackbody spectrum. The assumption of blackbody emission is justified, since for the shock velocity we infer, $v / c \approx 0.03$ (see Equation (4) below), there is no departure from thermal equilibrium. Such departure is expected only for higher velocity shocks, $v / c \gtrsim 0.1$ (Weaver 1976; Katz et al. 2010).

Based on Equation (1), and assuming that we are observing at wavelength $\lambda$ in the Rayleigh-Jeans tail,

$$
L_{\lambda} \approx \frac{v_{\mathrm{s}}}{c} L_{\lambda}^{\mathrm{BB}} \approx 4 \pi^{2} r_{\mathrm{s}}^{2} v_{\mathrm{s}} \frac{2 k_{B} T}{\lambda^{4}},
$$

where $L_{\lambda}^{\mathrm{BB}}$ is the blackbody total specific luminosity and $k_{B}$ is the Boltzmann constant.

By solving Equations (2) and (3), along with $r=v_{\mathrm{s}} t$, we find that

$$
v_{\mathrm{s}} \approx 1.3 \times 10^{4} L_{\lambda, 6 \mathrm{e} 38}^{1 / 3} \lambda_{6200}^{4 / 3} \kappa_{0.34}^{1 / 12} t_{7}^{-7 / 12} \mathrm{~km} \mathrm{~s}^{-1},
$$

where $L_{\lambda, 6 \mathrm{e} 38}$ is the specific luminosity in units of $6 \times$ $10^{38} \mathrm{erg} \mathrm{s}^{-1} \AA^{-1}$ (equivalent to the luminosity measured on 2009 June 23 , close to the first GALEX detection), $\lambda_{6200}$ is the wavelength at which the luminosity is measured in units of $6200 \AA, \kappa_{0.34}$ is the opacity in units of $0.34 \mathrm{~cm}^{2} \mathrm{~g}^{-1}$ (assuming the opacity of completely ionized solar composition), and $t_{7}$ is the time between the explosion and the measurement of $L_{\lambda}$, in units of 7 days. The distance from the star to the shell in which the breakout occurs is

$$
r_{\mathrm{s}}=v_{\mathrm{s}} t \approx 8.2 \times 10^{14} L_{\lambda, 6 \mathrm{e} 38}^{1 / 3} \lambda_{6200}^{4 / 3} \kappa_{0.34}^{1 / 12} t_{7}^{5 / 12} \mathrm{~cm} .
$$

This radius is larger than the size of a typical red supergiant, indicating that our assumption that the shock occurs "outside" the star, in a wind-density profile, is justified. The density is given by

$$
n=\frac{\rho_{\mathrm{s}}}{m_{\mathrm{p}}} \approx 4.8 \times 10^{10} L_{\lambda, 6 \mathrm{e} 38}^{-2 / 3} \lambda_{6200}^{-8 / 3} \kappa_{0.34}^{-7 / 6} t_{7}^{1 / 6} \mathrm{~cm}^{-3},
$$

where $m_{\mathrm{p}}$ is the proton mass, and we also have

$$
T \approx 9.1 \times 10^{4} \kappa_{0.34}^{-1 / 4} t_{7}^{-1 / 4} \mathrm{~K} .
$$

Here, $T$ is the effective temperature at the time in which we measured $L_{\lambda}$.

In addition, we can estimate the rise time of the SN light curve. In the case that the scale height of the material lying ahead of the shock is negligible compared with the radius at which the shock occurs $\left(r_{\mathrm{s}}\right.$, i.e., shock takes place at the edge of the star), the rise time is $\sim r_{\mathrm{s}} / c$ (up to a log correction factor mentioned earlier). However, in the scenario discussed here there is a significant amount of material ahead of the shock and the rise time is $t_{\text {rise }} \approx r_{\mathrm{s}} / v_{\mathrm{s}}=7 t_{7}$ day. This rise time is therefore slower than the rise time seen in some of the known shock-breakout events (e.g., Gezari et al. 2008). In this scenario, after maximum light, the light curve is expected to decay exponentially on the diffusion timescale $\left(t_{\text {diff }} ;\right.$ Falk \& Arnett 1977), which in our case is about a week. This is similar to the observed decay rate.

The total mass in the fast ejecta within radius $r_{\mathrm{s}}$ is $\int_{r_{*}}^{r_{\mathrm{s}}} 4 \pi r^{2} \rho(r) d r$. Here $r_{*}$ is the star radius. Assuming the wind density is $\rho(r) \propto r^{-2}$ and $r / r_{*} \gg 1$, we find

$$
M \approx 4 \pi \rho r_{\mathrm{s}}^{3} \approx 0.3 L_{\lambda, 6 \mathrm{e} 38}^{1 / 3} \lambda_{6200}^{4 / 3} \kappa_{0.34}^{-11 / 12} t_{7}^{17 / 12} M_{\odot} .
$$

Note that the total mass in the ejecta may be larger (see Section 3.2), and therefore this equation provides only a lower limit to the mass. Next, the mass-loss rate from the progenitor prior to the explosion is

$$
\dot{M} \approx \frac{M v_{w, 100}}{r} \approx 0.1 \kappa_{0.34}^{-1} t_{7} v_{w, 100}^{-1} M_{\odot} \mathrm{yr}^{-1},
$$

where $v_{w, 100}$ is the progenitor wind velocity in units of $100 \mathrm{~km} \mathrm{~s}^{-1}$. This mass-loss rate is required to persist for $r_{\mathrm{s}} / v_{w} \approx 10 \mathrm{yr}$ prior to the explosion. 
Another important property is the kinetic energy in the faster parts of the shock, which is roughly given by

$$
\begin{aligned}
E & \approx 4 \pi r_{\mathrm{s}}^{2} \frac{r_{\mathrm{s}}}{7} a T^{4} \approx \frac{4}{2} \pi r_{\mathrm{s}}^{3} \rho v_{\mathrm{s}}^{2} \\
& \approx 5 \times 10^{50} L_{\lambda, 6 \mathrm{e} 38} \lambda_{6200}^{4} \kappa_{0.34}^{-3 / 4} t_{7}^{1 / 4} \mathrm{erg} .
\end{aligned}
$$

As before the factor of 7 arises from the shock's compression ratio.

We note that if we relax the Rayleigh-Jeans approximation in Equation (3), and solve these equations using the full Planck formula, the solution does not change considerably. ${ }^{16}$ Therefore, for simplicity, we choose to show here the approximate solution.

Based on the temperature that we derive in Equation (7), the expected NUV luminosity is $2.1 \times 10^{40} \mathrm{erg} \mathrm{s}^{-1} \AA^{-1}$ (given by the Planck function multiplied by $v_{\mathrm{s}} / c$ ). This is about 1.5 times larger than observed. However, the UV emission may be affected by metal line blanketing, and is therefore less reliable than the visible-light luminosity.

Furthermore, we note that our assumption of a radiationmediated shock is justified since in the case studied here the ratio of radiation energy density to plasma thermal energy density is very large, $a T^{4} /\left(n k_{B} T\right) \approx 10^{6}$.

To summarize, based on our crude model we use the visiblelight luminosity to derive the shock properties (e.g., $r_{\mathrm{s}}, v_{\mathrm{s}}, T, E$, $M)$. The calculated $t_{\text {rise }}$ is roughly consistent with the observed rise time of the UV light curve. Moreover, this model naturally explains the high observed luminosity in the NUV band.

\subsection{The Bulk Velocity}

The velocity $v_{\mathrm{s}}$ we used so far corresponds to the faster parts of the ejecta. However, this does not necessarily represent most of the energy in the ejecta. As a sanity check, here we estimate the bulk velocity of the ejecta.

The shock that accelerates the ejecta gives more energy to slower shells. The energy as a function of velocity is given by

$$
E(v)=E_{\mathrm{b}}\left(\frac{v}{v_{\mathrm{b}}}\right)^{-x},
$$

where $E_{\mathrm{b}}$ is the bulk kinetic energy of the ejecta, $v_{\mathrm{b}}$ is the bulk velocity, and $x=5(1+3 n / 5) / n$, where $n=3 / 2$ (3), $x=19 / 3(14 / 3)$, for convective (radiative) envelopes ${ }^{17}$ (Matzner \& McKee 1999). A shell of velocity $v$ is decelerated when the energy in the shocked wind, $E_{w}$, equals the energy in the decelerated shell, $E(v)$. Here $E_{w}=4 \pi K r v^{2}$ and $E(v)=M(v) v^{2}$. Note that this includes the internal energy in the shocked wind (assuming the internal energy roughly equals the kinetic energy). This gives the deceleration radius

$$
r(v)=\frac{E_{\mathrm{b}}}{4 \pi K v_{\mathrm{b}}^{2}}\left(\frac{v}{v_{\mathrm{b}}}\right)^{-2-x} .
$$

The wind optical depth at this radius is $\kappa \rho r$ or

$$
\tau(v)=\frac{4 \pi \kappa K^{2} v_{\mathrm{b}}^{2}}{E_{\mathrm{b}}}\left(\frac{v}{v_{0}}\right)^{2+x} .
$$

As long as $\tau \gtrsim c / v_{\mathrm{s}}$, the fast shell is decelerated and overtaken by the slower, more energetic, shells behind it. Breakout occurs

\footnotetext{
16 The values of $v_{\mathrm{s}}, r_{\mathrm{s}}, t_{\text {rise }}$, and $M$ are changed by a factor of $1.04 ; n$ is changed by a factor of $0.92 ; E$ by 1.14 ; and $T$ and $\dot{M}$ remain the same. 17 In the notation of Matzner \& McKee (1999), $x=(n+1) /\left(n \beta_{1}\right)-2$, where $\beta_{1}=1 / 5$.
}

when $\tau(v) \approx c / v_{\mathrm{s}}$, which together with Equation (13) and assuming $E_{\mathrm{b}}=M_{\mathrm{b}} v_{\mathrm{b}}^{2} / 2$ gives

$$
\frac{v_{\mathrm{s}}}{v_{\mathrm{b}}}=\left(\frac{c}{v_{\mathrm{s}}} \frac{M_{\mathrm{b}}}{8 \pi \kappa K^{2}}\right)^{1 /(2+x)},
$$

where $M_{\mathrm{b}}$ is the total mass in the ejecta. Since $1 /(x+2) \ll 1$, the inferred value of $v_{\mathrm{s}} / v_{\mathrm{b}}$ is insensitive to the exact values of $v_{\mathrm{s}}$ and $K$, and to the unknown value of $M_{\mathrm{b}}$. Assuming $M_{\mathrm{b}}=1 M_{\odot}$, $v_{\mathrm{s}}=10^{4} \mathrm{~km} \mathrm{~s}^{-1}$, and $K=\dot{M} /\left(4 \pi v_{w}\right) \approx 5 \times 10^{16} \mathrm{~g} \mathrm{~cm}^{-1}$ (corresponding to $\dot{M}=0.1 M_{\odot} \mathrm{yr}^{-1}$ and $v_{w}=100 \mathrm{~km} \mathrm{~s}^{-1}$ ), we get $v_{\mathrm{s}} / v_{\mathrm{b}} \approx 1.1$ (1.2) for convective (radiative) envelopes.

This analysis suggests that Equations (4), (8), and (10) are reasonable approximations of the bulk properties of the ejecta. Specifically, if the total mass of the ejecta is an order of magnitude larger than that given by Equation (8), then the total kinetic energy of the SN will exceed $5 \times 10^{51} \mathrm{erg}$, which is unlikely (at least for the garden variety of $\mathrm{SNe}$ ).

\section{DISCUSSION}

We present the discovery of PTF 09uj, which was serendipitously observed by GALEX at early times after the explosion. The spectrum of the SN and the bright UV signal suggest that this was an SN IIn powered by the diffusion of the shock energy and interaction of the ejecta with a dense $\operatorname{CSM}\left(n \approx 10^{10} \mathrm{~cm}^{-3}\right)$. This interpretation is consistent with both the fast rise of the UV light curve and the UV luminosity. The observed fast rise cannot be easily explained unless the progenitor is embedded in an optically thick wind. Moreover, a shock breakout from a stellar photosphere cannot generate such a bright UV signal (e.g., Rabinak \& Waxman 2010).

Based on simple modeling, we suggest that prior to the explosion the progenitor went through a phase of high massloss rate, with $\dot{M} \approx 0.1 M_{\odot} \mathrm{yr}^{-1}$. The radius of the radiating region and the fast decay of the $\mathrm{SN}$ are suggestive of an episodic high mass-loss rate with a duration of about several years prior to the explosion. Our model suggests that the total mass of the ejecta is relatively low, roughly $1 M_{\odot}$. We stress that this is an order-of-magnitude estimate; the total ejected mass could be as high as a few solar masses, but probably not on the high end $\left(\gtrsim 10 M_{\odot}\right)$ of the ejected mass expected in typical SNe II.

The low ejecta mass, if true, may be due to one of the following: (1) most of the mass of the progenitor was not expelled by the SN explosion and it is in a form of a compact remnant or (2) most of the mass of the progenitor was expelled (e.g., wind) prior to the explosion. This is in contrast to more energetic SN explosions whose luminosity is powered by interaction with a dense CSM (e.g., Benetti et al. 2006; Ofek et al. 2007; Smith et al. 2007).

PTF is a wide and shallow survey in which about $3000 \mathrm{deg}^{2}$ are actively surveyed at a given time down to a limiting magnitude of $\sim 21$. GALEX sensitivity allows high signal-tonoise ratio detections of SN shock-breakout flashes at a redshift range which is similar to that probed by PTF. Specifically, we estimate that PTF should find several SNe each year for which GALEX early observations will be available. This estimate is based on the number of PTF SN discoveries, during 2009, which had GALEX serendipitous observations between 0 and 30 days prior to the PTF discovery.

We thank an anonymous referee for useful comments. E.O.O. and D.P. are supported by an Einstein fellowship. S.B.C. and A.V.F. acknowledge generous financial assistance from Gary \& 
Cynthia Bengier, the Richard \& Rhoda Goldman Fund, NASA/ Swift grants NNX09AL08G and NNX10AI21G, and NSF grant AST-0908886. A.G. acknowledges support by the Israeli and the US-Israel Binational Science Foundations, an EU/IRG fellowship, the Benoziyo Center for Astrophysics, and the Peter and Patricia Gruber Awards. The National Energy Research Scientific Computing Center, which is supported by the Office of Science of the U. S. Department of Energy under Contract No. DE-AC02-05CH11231, provided staff, computational resources, and data storage for this project. P.E.N. acknowledges support from the US Department of Energy Scientific Discovery through Advanced Computing program under contract DEFG02-06ER06-04. J.S.B.'s work on PTF was supported by NSF/ OIA award AST-0941742 ("Real-Time Classification of Massive Time-Series Data Streams"). L.B. and K.S. are supported by the NSF under grants PHY 05-51164 and AST 07-07633.

\section{REFERENCES}

Benetti, S., Cappellaro, E., Turatto, M., Taubenberger, S., Harutyunyan, A., \& Valenti, S. 2006, ApJ, 653, L129

Cenko, S. B., et al. 2006, PASP, 118, 1396

Cenko, S. B., et al. 2008, ApJ, 677, 441

Chugai, N. N., \& Danziger, I. J. 1994, MNRAS, 268, 173

Chugai, N. N., Danziger, I. J., \& Della Valle, M. 1995, MNRAS, 276, 530

Colgate, S. A. 1974, ApJ, 187, 333

Dessart, L., Hillier, D. J., Gezari, S., Basa, S., \& Matheson, T. 2009, MNRAS, 394,21

Falk, S. W., \& Arnett, W. D. 1977, A\&AS, 33, 515

Fassia, A., et al. 2000, MNRAS, 318, 1093
Fassia, A., et al. 2001, MNRAS, 325, 907

Filippenko, A. V. 1997, ARA\&A, 35, 309

Fransson, C., et al. 2002, ApJ, 572, 350

Gal-Yam, A., \& Leonard, D. C. 2009, Nature, 458, 865

Gal-Yam, A., Maoz, D., Guhathakurta, P., \& Filippenko, A. V. 2008, ApJ, 680 550

Gal-Yam, A., et al. 2004, ApJ, 609, L59

Gal-Yam, A., et al. 2007, ApJ, 656, 372

Gezari, S., et al. 2008, ApJ, 683, L131

Katz, B., Budnik, R., \& Waxman, E. 2010, ApJ, 716, 781

Klein, R. I., \& Chevalier, R. A. 1978, ApJ, 223, L109

Komatsu, E., et al. 2009, ApJS, 180, 330

Law, N. M., et al. 2009, PASP, 121, 1395

Martin, D. C., et al. 2005, ApJ, 619, L1

Matzner, C. D., \& McKee, C. F. 1999, ApJ, 510, 379

Miller, J. S., \& Stone, R. P. S. 1993, Lick Observatory Technical Reports 66 (Santa Cruz, CA: Lick Observatory)

Morrissey, P., et al. 2007, ApJS, 173, 682

Nakar, E., \& Sari, R. 2010, arXiv:1004.2496

Ofek, E. O., et al. 2007, ApJ, 659, L13

Piro, A. L., Chang, P., \& Weinberg, N. N. 2010, ApJ, 708, 598

Poznanski, D., Gal-Yam, A., Maoz, D., Filippenko, A. V., Leonard, D. C., \& Matheson, T. 2002, PASP, 114,833

Rabinak, I., \& Waxman, E. 2010, arXiv:1002.3414

Rau, A., et al. 2009, PASP, 121, 1334

Schawinski, K., et al. 2008, Science, 321, 223

Schlegel, D. J., Finkbeiner, D. P., \& Davis, M. 1998, ApJ, 500, 525

Schlegel, E. M. 1990, MNRAS, 244, 269

Smith, N., \& McCray, R. 2007, ApJ, 671, L17

Smith, N., et al. 2007, ApJ, 666, 1116

Smith, N., et al. 2009, ApJ, 695, 1334

Soderberg, A. M., et al. 2008, Nature, 453, 469

Waxman, E., Mészáros, P., \& Campana, S. 2007, ApJ, 667, 351

Weaver, T. A. 1976, ApJS, 32, 233

York, D. G., et al. 2000, AJ, 120, 1579 